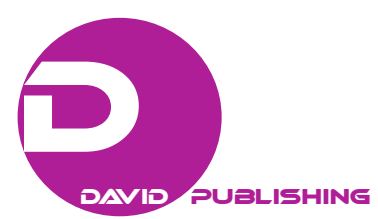

\title{
The Inclined Plane, Simple Machine and Calculus Geometric Register
}

\author{
Aouatif Najoua \\ University Hassan II, Mohammedia, Morocco \\ Jalila Achouaq Aazim \\ University Mohamed V, Rabat, Morocco \\ Mohamed Bahra, \\ CERMEF, Casablana, Morocco \\ Mohammed Talbi \\ University Hassan II, Casablanca, Morocco
}

\begin{abstract}
When two kinds of knowledge are nearby, the first one transposed into the school environment can constitute an obstacle to the proper functioning of the second without the professors realizing it. On the one hand this is the case for the notion of fractional number, and for the rational number or ratio. This teaching situation exemplifies the concept of the ecology of school knowledge (Chaachoua, 2007).
\end{abstract}

Keywords: inclined plane, school knowledge ecology, fraction, ratio

\section{Introduction}

Regarding the fractional calculus, with $m$ and $n$ as two natural integers and $n$ not null, we distinguish two operations types: 1) Operating on results, symbolized a fraction $\frac{m}{n}$, of the action that consists of subdividing an entity to a number $n$ of identical proportions and take a number $m$ from these proportions; 2) Operating on rationales, even on ratios. Normally, at the end of compulsory education, which is aimed at pupils (14-15 years of age), the first type of operation must give way to the second type, or at least a respectful niche that the latter should take. But it is very probable that the second type undergoes a strong domination of the first one until it is completely veiled in a large proportion of these pupils. It is also likely that this veil continues even among post-secondary students, including high-mathematics students.

Aouatif Najoua, assistant professor/doctorate, Laboratory of Signals, Distributed Systems and Artificial Intelligence, University Hassan II, Mohammedia, Morocco.

Jalila Achouaq Aazim, assistant professor/doctorate, Laboratory of Mechanics, Processes and Industrial Processes, University Mohamed V, Rabat, Morocco.

Mohamed Bahra, full professor/doctorate, Cell of bservation and Research in Teaching Science and Technology, CERMEF, Casablanca, Morocco.

Mohammed Talbi, full professor/doctorate, Research Observatory in Didactics and University Pedagogy, University Hassan II, Casablanca, Morocco.

Correspondence concerning this article should be addressed to Aouatif Najoua, ENSET, BP 159, Boulevard Hassan II, Mohammedia, Morocco. 


\section{Literature Review}

Studies on relationship between pupils and teachers with fractions and fractional calculus are numerous. Work of Rosar, Nieuwenhoven, and Jonnaert (2007) entitled Les fractions, comment mieux comprendre les difficultés rencontrées par les élèves? This is the typical example of these studies. These authors conclude their research with these terms:

...Indeed, would not it be better to spend more time exploring what a fraction, a number, before embarking on computing situations? Studying fractioning in its diversity without restricting it to the tart example, insisting on using ratio fractions, especially when working on a plane, a map, working on the fraction-number ordinal and cardinal properties, are some of the objectives on which the fractions learning should focus. Moreover, one can also wonder about the need to insist on certain complex operations (for example, dividing a fraction by another). Let us remember that these operations will be useful only when the student works algebra, probabilities, and powers. Why not concentrate mainly on operations used in these apprenticeships (addition, subtraction, and multiplication). (p. 13)

However, researches show how an institutional ecology favoring the veil of the ratio concept by fraction notion is established and persists are almost non-existent. On this institutional ecology notion, Bosch and Chevallard (1999) write:

Faced with situation in which a type of task proves to be problematic, an evolution and often even a progress appear when it is decided not to repress the problematicity of the task-an anthropologically most frequent situation, but to study the problem in the purpose of building the missing technique. This starts with a type of problematic tasks. For example, "How to solve a second degree equation?", "How to measure time?”, "How to count the number of people in a crowd?", and "How to introduce the decimal number notion?". If necessary, and after a longer or shorter study process, it is possible to produce techniques for providing answers to the questions initially asked. A new "know-how" is being built, which must still be organized to ensure it a regularly functioning in the institution. (p. 6 )

Moving from the fractioning task to work of magnitude or on commensurable magnitudes and on their commensurability is problematic: associated with a simple activity, the first is pregnant. On the other hand, not yet referring to an activity of such simplicity, the second faded before the first and struggled to make its way into the school environment and created a niche. Although it is the great pivot of mathematics, "know-how" relative to commensurability would be the object of a damaging dysfunction, as to the intelligibility of a whole fauna of mathematical concepts and unsuspected didactic practices.

\section{Research Methodology}

As the aforementioned dysfunction is a dysfunction in school knowledge ecology, methodology must be inspired by the institutional ecology constituting the Anthropological Theory of Didactics. According to Bosch and Chevallard (1999), we read it in the cited article:

... However, in any institutional routine, types of problematic tasks arise, for which no suitable technique exists, either because the task type is new (for the subject or for the institution) or because that the technique usually employed fails. There is then problem of constructing an adequate technique by adapting an old technique or by creating a new one. unless problem is simply denied and the appeared dysfunction is indefinitely prolonged, to the point of naturalizing it. (p. 6)

It is therefore a question of showing that problem of constructing adequate techniques, in terms of school institution, for carrying out addition and multiplication operations, not on results of an object fragmentation but on the commensurability of magnitudes, is a problem denied by this institution and that the underlying 
dysfunction is completely naturalized there. Perhaps in an institution other than school one this demonstration can be based on the existence of a task or practice by which achievement can be modeled by the two sought techniques. This task or practice, as it is known but never solicited by school institution, completes the demonstration of the veracity of negation and naturalization mentioned above.

With their manipulation, school practices make fractions of n-decimals, that is to say, two-decimals, three-decimals, etc., so that in the operation consisting of taking $2 / 3$ in a whole for instance, it is here about the three-decimal 0.2 , or 0.2 in the base 3 . We therefore deal each time with a single number, though in a different base, and not with a couple of natural integers.

It is therefore necessary to look for another practice and a task other than fragmentation or fractionation. The inclined plane can be this task.

Data of two natural integers $m$ and $n$ are sufficient to draw a rectangle on a grid: vertices of this rectangle are the grid nodes, one of these four sides is on a horizontal and counts $m$ squares, and another on a vertical and counts $n$ squares. Each rectangle constructed in this way corresponds to two natural integers $m$ and $n$. Thus, there is a correspondence between the set of pairs of natural integers and the set of these rectangles of the grid (the Cartesian notion of rectangular coordinates is a reorganization of this correspondence). Grid and this set of rectangles present themselves as candidates for constitution of substrate of the concept of ratio.

We consider the rectangles, where the summits are confused with grid nodes, and one of the sides has a support as one vertical. On the sides of a square as being the unit of length, we also admit that the ascending diagonal or the descending diagonal of such rectangle represents an inclined plane. In the case where the chosen diagonal is the ascending one, the denominator measures the duration made by a horizontal to go from the low horizontal side to the high horizontal side. This horizontal passes through a mobile point on the diagonal. Therefore, the ratio expresses the "rapidity" of the ascension of this mobile horizontal: It is sufficient to multiply by $m$ the length of vertical sides to obtain the representation of a rapidity $m$ times bigger and $n$ times the length of the horizontal sides to obtain that of a rapidity $n$ times smaller. The first operation consists in piling vertically $m$ times the same rectangle. The second is to pile horizontally $n$ times. Operation of multiplying two ratios and operation of adding two ratios are reduced, by means of arrangements of this type of stack, to operate on horizontal line ascent rapidities. These rapidities are represented by rectangles. These stacks thus constitute the substratum of a praxeology specific to the ratio concept.

To experiment this substrate, we think it would be convenient to make of it from the teaching environment of an ordinary lesson for first and second year of junior high school students and the epistemic environment for engineering student (junior high school students are solicited and observed as subjects). Engineering student is equally solicited, but the judges have to pronounce about the adequacy of this lesson to these students, they should first put themselves in their shoes and answer the questions asked.

The complete and specific description of the lesson and questions is presented in Appendix A, from the point of view of a solicitation text of the engineering students about the lesson. An example of students' productions is placed in Appendix $\mathrm{B}$, with an example of the point of view and productions of the engineering students.

The priori analysis of this same situation of junior high school student on the one hand and of engineering student on the other hand makes us consider the following two alternatives:

First alternative : 
A1-More than $75 \%$ of answers of junior high school student are correct;

A2-More than $25 \%$ of the learners' answers are incorrect.

Second alternative :

A'1-More than 25\% of the engineering students' answers are correct;

A'2-More than $75 \%$ of these answers are incorrect.

We consider that the verification of A1 signifies that the implicated knowledges in the questions of the lessons are situated in the natural extension of the knowledges officially due to the students. In virtue of what has been discussed above, the fact that these knowledges are not explicitly part of the officially due ones is an argument in favor of the coming conclusion C1: It is possible to prevent the second type of operation mentioned in the introduction from undergoing domination of the first type so that it would not be veiled by it in a large proportion of these students.

We also consider that the verification of A'2 (the second alternative) is an argument in favor of the following conclusion C2: It is very probable that the second type undergoes a strong domination of the first one until it completely veils it in a large proportion of junior high school student. It is also likely that this veil continues even among post-secondary students, even among high-mathematics students.

On the other side, if it is A2 that occurs repeatedly in the first alternative then conclusion C1 loses any argument in its favor. It is the same for the conclusion C2, if it is A'1 which is confirmed in the second alternative.

\section{Results}

The questions answered by the tested students are equally important. They are not subject to any hierarchy. In addition, the number of students' answers is important, and so is the proportion of correct answers. We have received 204 answers from 34 students of junior high school for six questions that we have asked. The non-answers are counted as wrong answers. In these 204 answers, 183 are correct, which means $89.7 \%$ of the answers.

We have received 1,344 answers from 224 solicited engineering students to put themselves in the place of students and to answer six questions. The non-answers are counted as wrong answers. In these 1,344 answers, only 58 are correct, which means $4.31 \%$ of the answers.

\section{Analysis or Discussion}

The correct answers represent $89.7 \%$ of the answers of the junior high school students. This proportion largely crosses the threshold of the fixed $75 \%$ according to the adopted methodology. Therefore, it would be possible to make of the inclined plane, associated with the para-mathematical and para-physical notion of rapidity of ascension or descent of the horizontal line, as explained above, the pivot of the contextualization of the ratio concept. There is also a certain guarantee of success of integrating knowledge on the fractional calculus, already acquired by the students, through this contextualisation to knowledge related to this concept.

Among other notions, it is obvious that the notions of similar triangles, theorem of Thales, and the vector-speed concept can be grafted onto this notion of rapidity of ascension. This possibility of grafting confirms the inclined plane in its role of precursor of an ecological niche for the ratio concept and of commensurability. If we consider that the inclined plane is the only one capable of assuming this role, then the fact that it is always a blind spot of successive, didactic relations that the school practices build around fraction 
and ratio concepts tends to confirm the negation and naturalization mentioned in the citation about the methodology. This fact also appears as a strong argument in favor of conclusion C1.

The correct answers are not only representing $4.31 \%$ of the engineering students' answers. This proportion is so far from the threshold of $25 \%$ fixed according to the adopted methodology. We conclude the failing of the inclined plane to be perceived by the engineering students as capable to be placed with profit to the principal of the contextualization of ratio concept. Therefore, the relative knowledges of this concept do not find an anchoring point in the nearby knowledges already acquired by the engineering students. This is a very strong argument in favor of the conclusion stipulating that the hypothesis $\mathrm{H}$ is a wrong hypothesis.

\section{Conclusion}

In view of the answers of college pupils and tested engineering students, it can be said that fraction notion constitutes an obstacle to the formation of a viable niche in the school environment for the concept of ratio. A manipulation of rectangular shapes in the grid shows that the inclined plane may well be the substrate of a didactic medium precursor of the formation of this niche. Everything happens as if the predominance of the inclined plane subordination (well-known as a simple machine) prevents the emergence of the assignment of the role of precursor of the niche in question to this plane through a manipulation of such didactic practices. Provided that the inclined plane is the sole substrate of such an environment, it follows that the formation of the niche in question will not see the light as long as the school practices subjected to this pregnancy remain blind to this manipulation.

\section{References}

Bosch, M., \& Chevallard, Y. (1999). La sensibilité de l'activité mathématique aux ostensifs. Recherches en didactique des mathématiques, 19(1), 77-124.

Chaachoua, H. (2007). Master2 R et P, IC2A, Didactique des Sciences UE TC2. Concepts et méthodes en didactique des sciences. Cours «Théorie anthropologique». Retrieved from http://imss-www.upmf-grenoble.fr/prevert/SpecialiteDEMS/Cours\%202007/UE2/UE2La\%20proble\%CC\%81matique\%20e \%CC\%81cologique_2007.pdf

Chevallard, Y. (2006). La théorie anthropologique des faits didactiques devant l'enseignement de l'altérité culturelle et linguistique Le point de vue d'un outsider. DOCPLAYER. Retrieved from http://yves.chevallard.free.fr/spip/spip/IMG/pdf/La_TAD_devant_l_alterite_culturelle_et_linguistique.pdf

Rosar, D., \& Nieuwenhoven, C. V. (2001). Les fractions, comment mieux comprendre les difficultés rencontrées par les élèves? Instantanés $\quad$ Mathématiques, $37(2), \quad$ 4-17. $\quad$ Retrieved from https://cudc.uqam.ca/upload/files/module/Les_fractions_comment_mieux_comprendre_les_difficultes_rencontrees_par_les_ eleves.pdf 


\section{Appendix A}

\section{The solicitation of the point of view of the engineer-students about the teaching question:}

The mathematics of the engineer often brings quantitative knowledges into play, making the ratio concept work. Thus, in the primary education and even the junior high school, the fraction concept is constituted in a trial to ratio concept. Therefore, the engineer-students are the well-placed to perceive retrospectively this trial. In the following, we present an activity destined to junior high school students. This activity is supposed to carry out crossing the trial in question. We await that the engineer-students put themselves in the place of the student (read the presentation and answer the questions just as a junior high school student will do), then to give their point of view about the relevance and the opportunity of introducing this activity in the curricula of junior high school education, around the world.

Here is the title and the plane of the lesson, just as they are suggested to junior high school teachers who accepted to make the experimentation.

\section{Title : Fractional calculus technics and expression technics in French}

Lesson objectives

Show that we can control the technics of fractional calculus without controlling the expression technics in French (or Arabic or Berber etc.) about the words "rapidity and slowness".

\section{Activity presentation}

About what:

Let us consider a rectangle $A B C D$ in the grid with the segment $[A B]$ in a horizontal, with $B$ in the right of $A$; and [CD] in a horizontal, with $\mathrm{D}$ above the $\mathrm{A}$. We take $\mathrm{AB}=5$ squares and $\mathrm{AD}=3$ squares $\rightarrow$ Who can do this?

\section{The expression technics at first :}

To develop clear expressions about the word "rapidity" using this rectangle, we need two coordinated straight lines in each of the two diagonals of the rectangle $\rightarrow$ (who can show me the two diagonals?):

- a vertical straight line, slipping horizontally from left to right, which we will represent the chronometer with (to talk about the rapidity we need a time measurer)

- an horizontal straight line, slipping vertically from high to low or from low to high according to the chosen diagonal, which we will represent the elevator with (the elevator goes up and down more or less rapidly)

Using the rectangle $\mathrm{ABCD}$, how we can constitute the two straight lines is independent of the other and how can we constitute it? How to constitute the dependent one while the diagonal [AC] is assuring the coordination?

Answer:

The chronometer straight line is the independent straight line when it is perpendicular to the side $[\mathrm{AB}]$ of the rectangle in point $\mathrm{T}$. The point $\mathrm{T}$ slips from A to $\mathrm{B}$ at a rate of a square per second. The elevator straight line is the dependent straight line when it is the diagonal [AC], which assures the coordination between the independent straight line and the dependent straight line. The elevator straight line is perpendicular to the chronometer straight line in the point where this straight line cuts this diagonal.

\section{Questions:}

In the case where the diagonal [AC] which assures the coordination, completes the blanks in the coming lacunar expression:

When the chronometer straight line crosses from (AD) to (BC), .....passes and the elevator straight line... from... crossing from $(\ldots)$ to $(\ldots)$.

In the case where the diagonal [DB] which assures the coordination, completes the blanks in the coming lacunar expression: When the chronometer straight line crosses from (AD) to (BC), .....passes and the elevator straight line... from... crossing 
from (...) to (...).

To mobilize the expressions "in”, “for each”, “all the”, “at a rate of... by”, and "with a rapidity of”, complete the blanks in the coming sentences knowing that it is the diagonal [AC] which assures the coordination:

The elevator straight line... from.... in...

The elevator straight line... from... for each... passed

The elevator straight line... from... all the ...

The elevator straight line... at a rate of... by...

The elevator straight line... with a rapidity of $\frac{3}{5}$ square/second

To mobilize the expressions "in”, “for each”, “all the”, "at a rate of... by”, and “ with a rapidity of”, complete the blanks in the coming sentences knowing that it is the diagonal [BD] which assures the coordination:

The elevator straight line... from... In...

The elevator straight line... from... for each... passed

The elevator straight line... from... all the...

The elevator straight line... at a rate of... by...

The elevator straight line... with a rapidity of $\frac{3}{5}$ square/second

For each of the traced figures, indicate, expressing by a fraction, the represented rapidity (the teacher suggests as figures rectangles, each one with one of the two traced diagonals).

Starting from the rectangle and the diagonal representing $\frac{5}{4}$ square/second, constitute, as a disposition, in dominos, many rectangles:

The rectangle and the diagonal representing the rapidity which is two times lower;

The rectangle and the diagonal representing the rapidity which is three times bigger;

The rectangle and the diagonal representing the rapidity which is three times bigger than which is two times less. 


\section{Appendix B: Copy a 6th grade junior high school student}

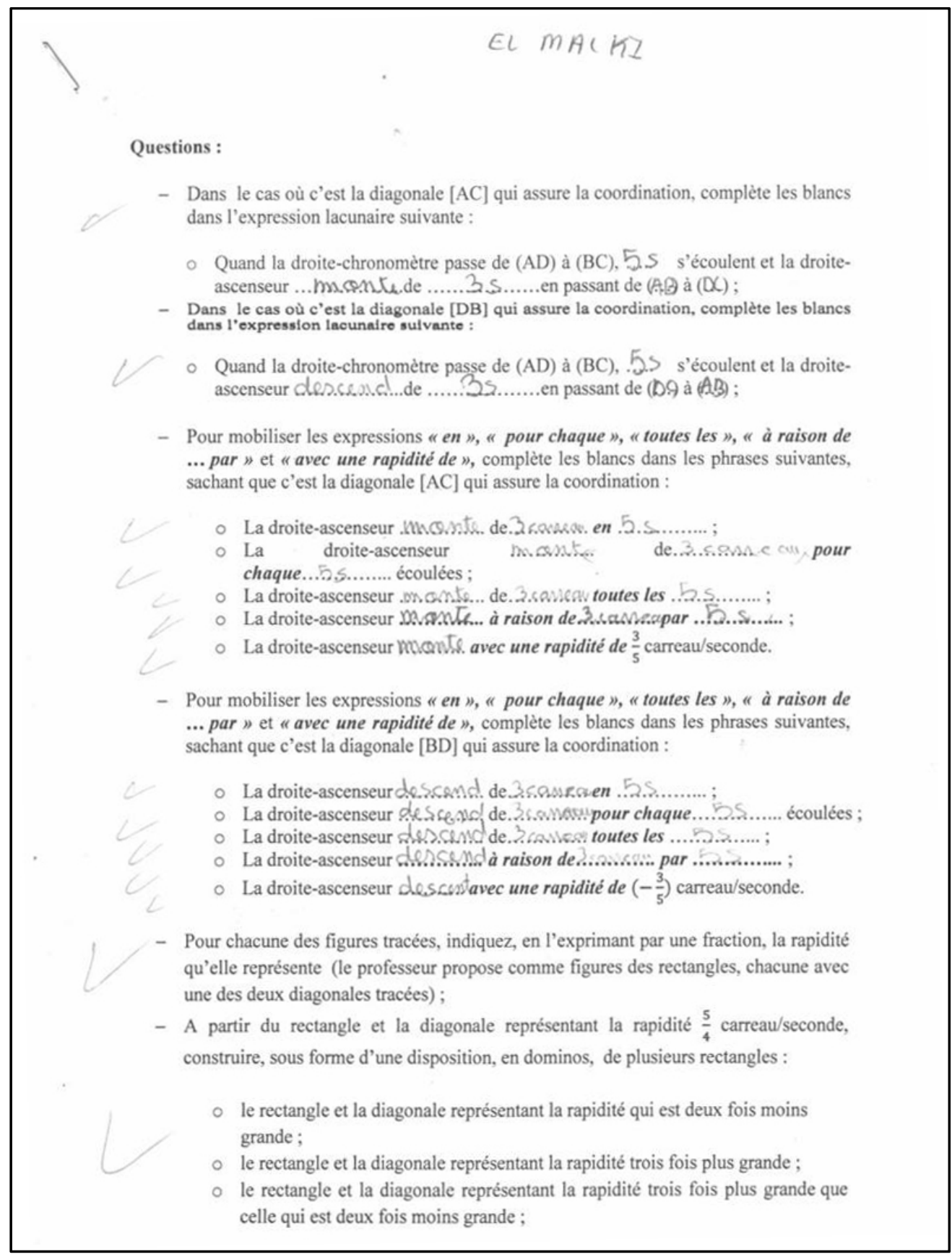

Questions:

In the case where the diagonal [AC] which assures the coordination, complete the blanks in the coming lacunar expression: When the chronometer straight line crosses from (AD) to (BC), $5 \mathrm{~S}$ passes and the elevator straight go up from $\mathbf{3} \mathrm{S}$ crossing from (AB) to (DC).

In the case where the diagonal [DB] which assures the coordination, complete the blanks in the coming lacunar expression: When the chronometer straight line crosses from (AD) to (BC) $5 \mathrm{~S}$ passes and the elevator straight line come down from $3 \mathrm{~S}$ 
crossing from (DC) to (AB).

To mobilize the expressions "in”, "for each”, “all the”, "at a rate of... by”, and "with a rapidity of”, complete the blanks in the coming sentences knowing that it is the diagonal [AC] which assures the coordination:

The elevator straight line goes up from 3 squares in 5 squares

The elevator straight line goes up from 3 squares for each 5 seconds passed

The elevator straight line goes up from 3 squares all the 5 seconds

The elevator straight line goes up at a rate of $\mathbf{3}$ squares by 5 seconds

The elevator straight line goes up with a rapidity of $\frac{3}{5}$ square/second

To mobilize the expressions "in”, “for each”, “all the”, “at a rate of... by”, and "with a rapidity of”, complete the blanks in the coming sentences knowing that it is the diagonal [BD] which assures the coordination:

The elevator straight line goes down from $\mathbf{3}$ squares in $\mathbf{5}$ seconds

The elevator straight line goes down from $\mathbf{3}$ squares for each $\mathbf{5}$ seconds passed

The elevator straight line goes down from 3 squares all the $5 \mathrm{~S}$

The elevator straight line goes down at a rate of $\mathbf{3}$ squares by $\mathbf{5} \mathrm{S}$

The elevator straight line goes down with a rapidity of $\frac{3}{5}$ square/second

For each of the traced figures, indicate, expressing by a fraction, the represented rapidity (the teacher suggests as figures rectangles, each one with one of the two traced diagonals).

Starting from the rectangle and the diagonal representing $\frac{5}{4}$ square/second, constitute, as a disposition, in dominos, many rectangles:

The rectangle and the diagonal representing the rapidity which is two times lower;

The rectangle and the diagonal representing the rapidity which is three times bigger;

The rectangle and the diagonal representing the rapidity which is three times bigger than which is two times less.

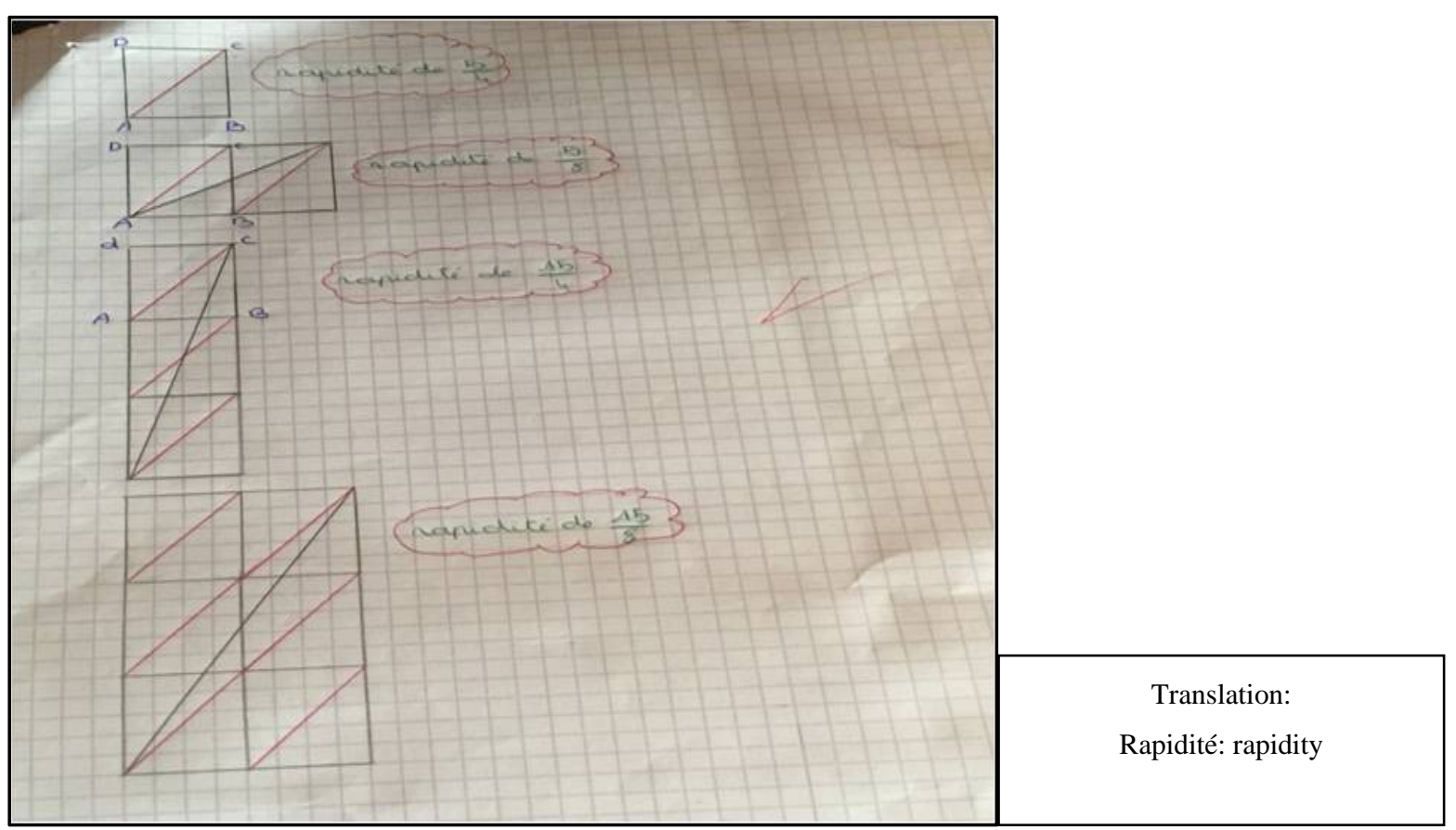

Engineer student copy: Translation of responses. 
In the case where the diagonal [AC] which assures the coordination, complete the blanks in the coming lacunar expression:

When the chronometer straight line crosses from (AD) to (BC), seconds passes and the elevator straight translate from bottom up from $3 \mathrm{~S}$ crossing from (AB) to (DC);

In the case where the diagonal $[\mathrm{DB}]$ which assures the coordination, complete the blanks in the coming lacunar expression:

When the chronometer straight line crosses from (AD) to (BC) second passes and the elevator straight line slids from top to bottom from crossing from (BC) to (AB);

To mobilize the expressions "in”, "for each”, “all the”, "at a rate of ... by”, and "with a rapidity of”, complete the blanks in the coming sentences knowing that it is the diagonal [AC] which assures the coordination:

The elevator straight line translates from bottom up crossing from (AB) to (CD)

The elevator straight line goes up from 3 squares for each 5 seconds passed

The elevator straight line goes up from 3 squares all the 5 seconds

The elevator straight line translates at a rate of $\mathbf{3}$ squares by $\mathbf{5}$ seconds

The elevator straight line goes up with a rapidity of $\frac{3}{5}$ square/second

To mobilize the expressions "in”, “for each”, “all the”, “at a rate of... by”, and "with a rapidity of”, complete the blanks in the coming sentences knowing that it is the diagonal [BD] which assures the coordination:

The elevator straight line goes down crossing from (AD) to (AB)

The elevator straight line goes down from $\mathbf{3}$ squares for each $\mathbf{5}$ seconds passed

The elevator straight line goes down from 3 squares all the $5 \mathrm{~S}$

The elevator straight line goes down at a rate of $\mathbf{3}$ squares by $5 \mathrm{~S}$

The elevator straight line goes down with a rapidity of $\frac{3}{5}$ square/second

Opinion of engineer student

\section{Establishment: ENSET}

Option: Industrial Logistic Management

Level: 1st year

Opinion:

This is incomprehensible by the students of junior high school secondary.

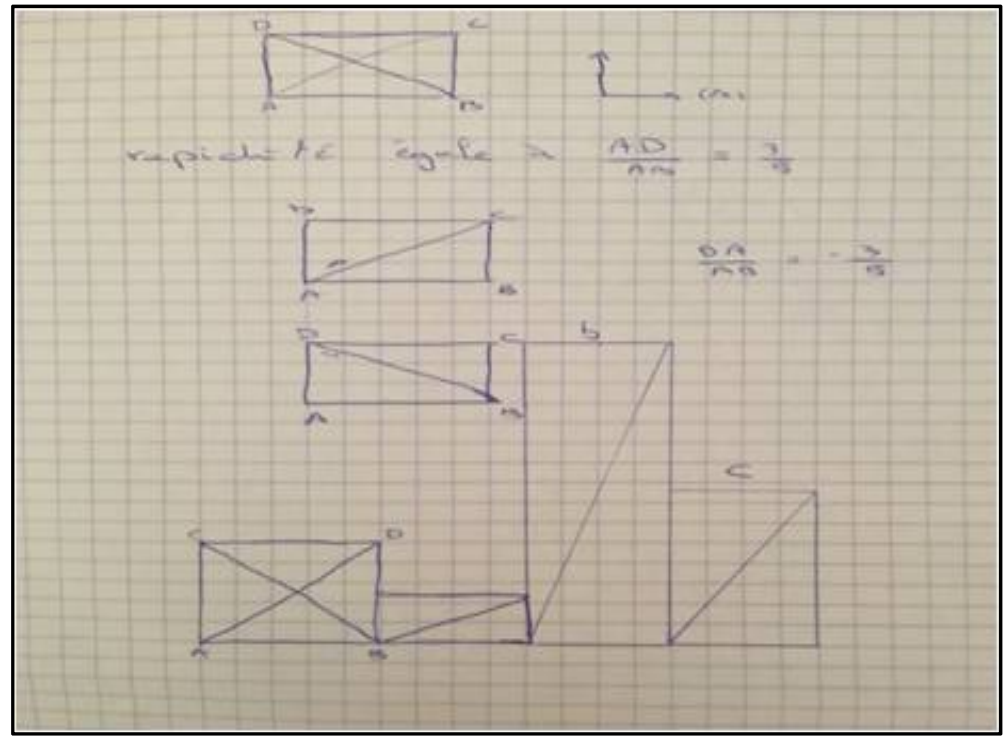

